\title{
Influence of Bridge Deck Pavement and Hinge Joints Damage on Transverse Load distribution of Void Slab Bridge
}

\author{
LIU Junli, a, JING Tianhu, ${ }^{2, b}$,Qiu Tiaoyuan ${ }^{1, c}$ \\ ${ }^{1}$ Guilin University of Technology, Guilin 541004, Guangxi P.R. China \\ ${ }^{2}$ Guangxi Key Laboratory of Geomechanics and Geotechnical Engineering, Guilin 541004, \\ Guangxi P.R. China \\ a1208078984@qq.com, b908801689@qq.coml, c1002284221@qq.com
}

\begin{abstract}
Keywords: void slab bridge; bridge deck pavement; hinge joint damage; transverse distribution coefficient
\end{abstract}

Abstract. In order to study the influence of bridge deck pavement and hinge joints on transverse load distribution of void slab bridge, this thesis took actual engineering as background and applied the finite element software ANSYS to establish a finite element analysis model of simply-supported void slab bridge. By changing the thickness of deck pavement layer and the extent of hinge joints damage, this thesis analyzed transverse distribution characteristics of load of simply supported void slab bridge. The results showed that the extent of hinge joints damage has larger influence on transverse distribution of void slab bridge load than the thickness of pavement layer. What's more, within a certain range, increasing the thickness of pavement layer can improve the load transverse distribution of the structure.

\section{Introduction}

Precast void slab bridges take a large proportion of small and medium span bridges in China. Transverse stiffness of deck pavement, hinge joints and other structures of void slab bridge enable vehicle load spread along the vertical and horizontal directions simultaneously, and each piece of primary beam will work in varying degrees, and the transverse distribution coefficient of load reflects the contribution ratio of each piece of beam. In actual engineering, the bridge deck pavement and primary beam of void slab form a superposition structure, which has a certain contribution to the carrying capacity of void slab bridge ${ }^{[1]}$.

However, during the service of void slab bridge, due to aging structures and overloaded vehicles, hinge joints of void slab bridge are damaged in varying degrees, and the transverse linkage is weakened, and transverse distribution coefficient of load will change, resulting in "single slab carrying load", thus changing the load scheme of the primary beam. In recent years, among bridge collapse caused by overloaded vehicles, fabricated void slab bridges whose single slab carries the load account for a large proportion $^{[2]}$, such as approach spans of third bridge of the Qiantang River Bridge and Zhengzhou City Zhongzhou Avenue across Beijing-Guangzhou Railway Bridge. Study on effect of pavement layer and extent of hinge joints damage on transverse distribution coefficient of void slab bridge load provides guidance on design, evaluation and reinforcement of prefabricated void slab bridge.

This thesis applied the finite element method to undertake solid finite element simulation of simply supported single span void slab bridge, adjusted the thickness of the pavement layer and the grade of hinge joints damage, and calculated the transverse distribution coefficient of void slab bridge load under different circumstances, providing certain reference to the evaluation and reinforcement of old bridges.

\section{Calculation methods of transverse distribution of load}

Calculation methods of transverse distribution of load that is suitable for void slab bridge mainly include: hinge slab method, space grillage method and solid finite element method.

The hinge slab method is more appropriate to prefabricated void slab bridge made of cast-in-place concrete which uses longitudinal tongue and groove joint. The calculation assumes that the hinge joints 
only transfer vertical shear, ignoring shear longitudinal to the bridge, normal force and moment transverse to the bridge. The calculation process is simple and it's easy to look up table and calculate manually. But it ignores the actual stiffness of hinge joints, and transverse connection only considers the role of hinge between the slabs rather than the combined effect of bridge deck pavement. Space grillage method is applicable to the plate-type, beam slab-type and box girder-section bridge structures and is effective to analyze especially curved and skew beam bridge ${ }^{[3-5]}$. Grillage method has clear concept, which is easy to understand and use, so it' s a practical and effective method to analyze the upper structure of bridge, but it also ignores the stiffness of hinge joints and assisting force of concrete of bridge deck pavement layer.

Solid finite element method disperses structure into three-dimensional solid element and can simulate various complex section members and connection among them. It can also fully consider the influence of warping, shear lag and distortion ${ }^{[6]}$. Three-dimensional solid finite element method can be used to accurately simulate the actual situation of the bridge structure, thereby studying the influence pattern of pavement layer thickness and hinge joint damage extent on transverse distribution coefficient of bridge load.

Based on reality, this thesis used the finite element software ANSYS to establish a solid structure model of void slab bridge, and used solid finite element method to study the influence pattern of pavement layer thickness and hinge joint damage extent on transverse distribution of structural load.

\section{Finite element analysis model}

Project overview.The calculation and analysis object is fabricated simple supported void slab bridge with span $1=20 \mathrm{~m}$. The section size of side slab and medium slab and cross-sectional arrangement are shown in Figure 1 and 2, respectively.

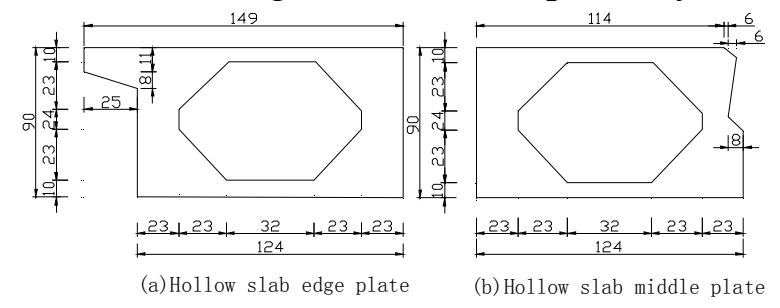

Figure 1 Section size of side slab and medium slab (unit: $\mathrm{cm}$ )

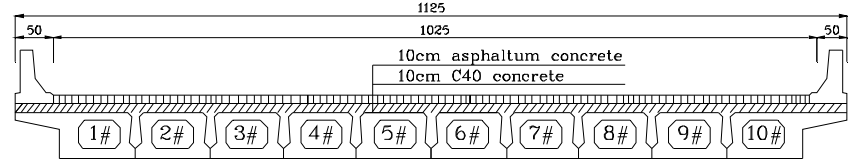

Figure 2 Section arrangement of void slab bridge (unit: $\mathrm{cm}$ )

Structural modeling. It uses solid unit Solid45 in ANSYS' s unit to simulate concrete. The unit forms hexahedral unit through eight nodes, each of which has three translational degrees of freedom along the $\mathrm{X}, \mathrm{Y}$ and $\mathrm{Z}$ direction. The unit has plasticity, creep, expansion, stress stiffening, large deformation and large strain capacity. The model uses hexahedral mapping to divide mesh, whose size is controlled at $0.2 \mathrm{~m}$. Bridge abutment is constrained with simple support.

According to the analysis requirements, considering the influence pattern of pavement layer thickness and hinge joints on transverse load distribution coefficient, the thesis established 10 models with pavement layer thicknesses of $5 \mathrm{~cm}, 10 \mathrm{~cm}, 15 \mathrm{~cm}, 20 \mathrm{~cm}$ and extent of hinge joints of $0,50 \%, 70 \%$, $80 \%, 90 \%, 100 \%$, respectively (Figure 3), wherein the extent of hinge joints damage is achieved by stiffness reduction.

Loading method. Based on analysis requirements, the loading method applies unit force, and it is arranged according to maximum moment of mid-span along the longitudinal direction. The analysis considers two operating modes when imposing unit force on side slab and medium slab, and studies the variation of transverse distribution characteristics in response to changes of pavement layer thickness and damage extent of hinge joints. The loading position is shown in Figure 4. 


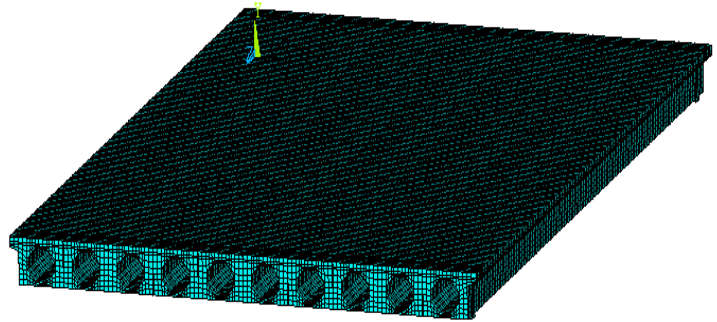

Figure 3 Spatial solid finite element model of void slab bridge

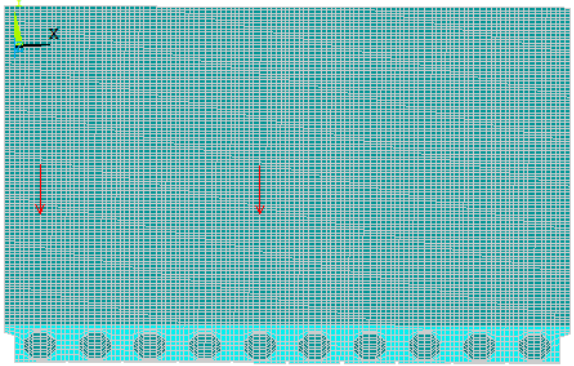

Figure 4 Loading position

\section{Result and analysis of transverse distribution coefficient}

Calculation results. This thesis mainly analyzed transverse distribution characteristics of void slab under the action of unit load $(1 \mathrm{kN})$. All nine hinge joints were undertaken elastic modulus reduction to simulate the damage. Transverse distribution coefficient is equivalent to transverse distribution influence line, so the thesis applied transverse distribution influence line as the index.

Thickness of deck pavement. Table 1 and 2 show variation of ordinate value of bridge transverse influence line with changes of pavement layer thickness when hinge joints of void slab are in good condition and imposing unit load on \#1 (side slab) and \#5 (medium slab) respectively.

It can be found from Table 1that with the increasing of pavement layer thickness, influence line value of slab \#1 shows a decreasing trend, indicating that the pavement layer can allocate load more evenly to other void slabs and bridge transverse distribution effect is enhanced. Figure 5 gives the proportion of mid-span deflection of slabs when imposing unit load on the side slabs.

Table 1 Transverse influence line values of slabs under different pavement layer thicknesses

\begin{tabular}{lllll}
\hline Slab & \multicolumn{4}{l}{ Thickness of deck pavement } \\
No. & $5 \mathrm{~cm}$ & $10 \mathrm{~cm}$ & $15 \mathrm{~cm}$ & $20 \mathrm{~cm}$ \\
\hline 1 & $\mathbf{2 3 9}$ & $\mathbf{2 3 7}$ & $\mathbf{2 3 5}$ & $\mathbf{2 3 3}$ \\
2 & 195 & 194 & 192 & 191 \\
3 & 153 & 153 & 152 & 151 \\
4 & 119 & 118 & 118 & 118 \\
5 & 90 & 91 & 91 & 91 \\
6 & 69 & 69 & 70 & 70 \\
7 & 51 & 52 & 53 & 53 \\
8 & 38 & 39 & 39 & 40 \\
9 & 27 & 28 & 30 & 31 \\
10 & 19 & 20 & 21 & 22 \\
\hline
\end{tabular}

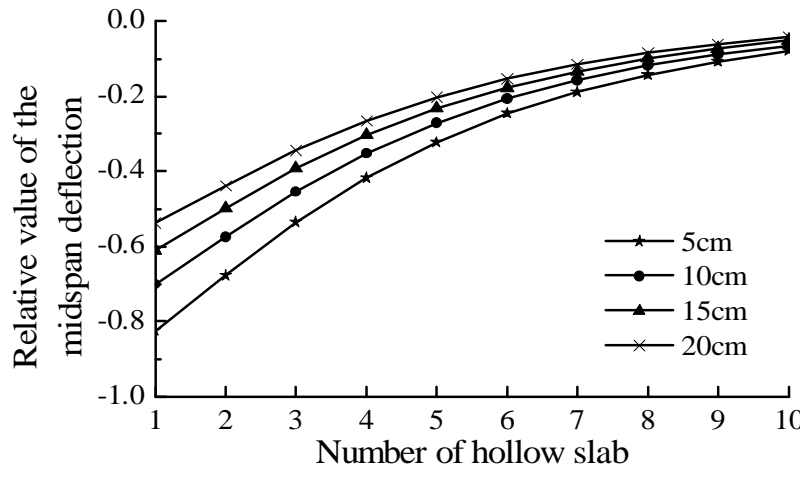

Figure 5 Pavement layer-proportion of mid-span deflection of void slabs

It can be seen from Figure 5 that with the increasing of pavement layer thickness, the deflection curve of void slab \#1 tends to be leveled off, that is, transverse coefficient decreases as the thickness increases. The coefficient of slab \#1 reduces most obviously--the deflection reduces by $37.0 \%$ when the pavement layer thickness is $20 \mathrm{~cm}$ compared to $5 \mathrm{~cm}$, indicating increasing pavement layer thickness can enhance transverse distribution effect and effectively improve the bending strength of void slab.

It can be found from Table 2 that with the increase of pavement layer thickness, the influence line value of slab \#5 still shows a decreasing trend, but in a relatively low speed, so side slab plays a major role in the calculation of transverse distribution coefficient. The following Figure 6 shows the proportion of mid-span deflection of slabs when imposing unit load on the medium slabs.

It can be seen from Figure 6 that bridge deflections are obviously different with different pavement layer thicknesses. The deflection of slab \#5 decreases by $36.3 \%$ when the pavement layer thickness is $20 \mathrm{~cm}$ compared to $5 \mathrm{~cm}$, indicating that with the increase of pavement layer thickness, the overall stiffness of the bridge increases and deflection decreases; at the same time, with the increase of pavement layer thickness, the deflection proportion curve slows down, indicating that the load is 
evenly distributed to each void slab. It shows that increasing pavement layer thickness can improve load capacity of simply supported void slab bridges.

Table 2 Transverse influence line values of slabs under different pavement layer thicknesses

\begin{tabular}{ccccc}
\hline $\begin{array}{c}\text { Slab } \\
\text { No. }\end{array}$ & $5 \mathrm{~cm}$ & \multicolumn{3}{c}{ Thickness of deck pavement } \\
\hline 1 & 92 & 92 & 92 & $15 \mathrm{~cm}$ \\
2 & 100 & 100 & 100 & $20 \mathrm{~cm}$ \\
3 & 109 & 109 & 109 & 100 \\
4 & 119 & 119 & 119 & 119 \\
5 & $\mathbf{1 2 3}$ & $\mathbf{1 2 3}$ & $\mathbf{1 2 3}$ & $\mathbf{1 2 2}$ \\
6 & 116 & 116 & 116 & 115 \\
7 & 103 & 103 & 102 & 103 \\
8 & 90 & 90 & 90 & 90 \\
9 & 78 & 79 & 79 & 79 \\
10 & 69 & 70 & 70 & 71 \\
Total & 1000 & 1001 & 1000 & 1000 \\
\hline
\end{tabular}

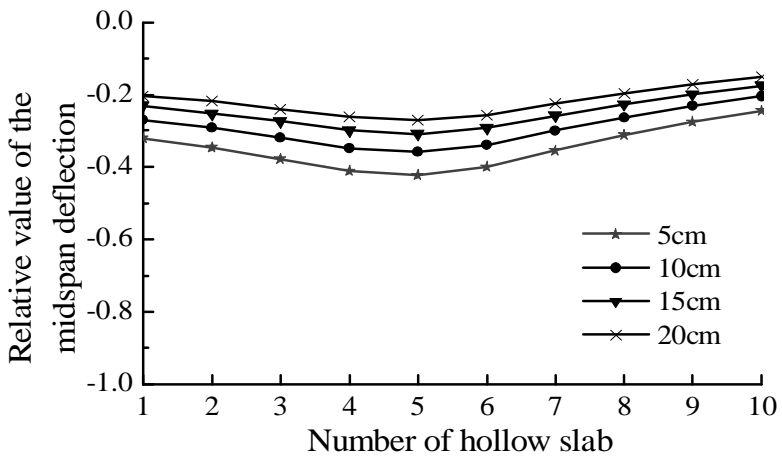

Figure 6 Pavement layer-proportion of vertical deflection of void slabs

Extent of hinge joints damage. Table 3 and 4 show variation of ordinate value of bridge transverse influence line with changes of extent of hinge joints damage when there is no pavement layer in void slab and imposing unit load on \#1 (side slab) and \#5 (medium slab) respectively.Impose unit load on side slab.

Table 3 Transverse influence line values of slabs with different extents of hinge joints damage

\begin{tabular}{ccccccc}
\multicolumn{7}{c}{ extents of hinge joints damage } \\
\hline Slab & \multicolumn{6}{c}{ Extent of hinge joints damage } \\
No. & None & $50 \%$ & $70 \%$ & $80 \%$ & $90 \%$ & $100 \%$ \\
\hline 1 & $\mathbf{2 0 0}$ & $\mathbf{2 0 2}$ & $\mathbf{2 0 4}$ & $\mathbf{2 0 6}$ & $\mathbf{2 1 9}$ & $\mathbf{2 3 8}$ \\
2 & 170 & 172 & 173 & 175 & 183 & 194 \\
3 & 142 & 142 & 143 & 144 & 148 & 152 \\
4 & 117 & 117 & 117 & 118 & 119 & 118 \\
5 & 96 & 96 & 96 & 96 & 94 & 90 \\
6 & 80 & 79 & 78 & 78 & 74 & 68 \\
7 & 65 & 64 & 64 & 63 & 58 & 51 \\
8 & 53 & 52 & 52 & 50 & 45 & 39 \\
9 & 43 & 42 & 41 & 40 & 34 & 29 \\
10 & 34 & 34 & 33 & 31 & 25 & 22 \\
Total & 1000 & 1000 & 1001 & 1001 & 999 & 1001 \\
\hline
\end{tabular}

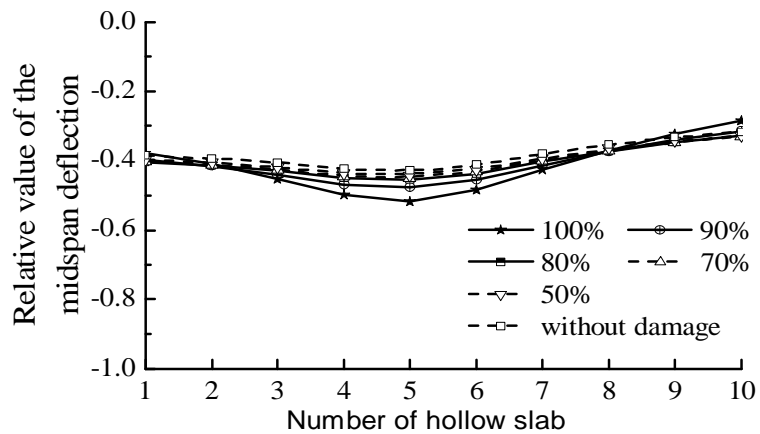

Figure 7 Extent of hinge joints damage-proportion of vertical deflection of void slabs

It can be found from Table 3 that with the increase of the extent of hinge joints damage, influence line values of \#1 4 slabs tend to increase, while when the hinge joints damage reaches 80\% 100\%, influence line value of slab \#1 increases sharply. Figure 7 shows the proportion of mid-span deflection of slabs when imposing unit load on the side slabs.

Figure 4 Transverse influence line values of slabs with different extents of hinge joints damage

\begin{tabular}{ccccccc}
\hline Slab & \multicolumn{6}{c}{ Extent of hinge joints damage } \\
No. & None & $50 \%$ & $70 \%$ & $80 \%$ & $90 \%$ & $100 \%$ \\
\hline 1 & 101 & 101 & 100 & 100 & 97 & 91 \\
2 & 103 & 103 & 103 & 102 & 102 & 99 \\
3 & 106 & 106 & 106 & 106 & 107 & 109 \\
4 & 111 & 111 & 111 & 112 & 115 & 120 \\
5 & $\mathbf{1 1 2}$ & $\mathbf{1 1 2}$ & $\mathbf{1 1 2}$ & $\mathbf{1 1 3}$ & $\mathbf{1 1 6}$ & $\mathbf{1 2 5}$ \\
6 & 107 & 108 & 108 & 108 & 111 & 117 \\
7 & 99 & 99 & 100 & 100 & 101 & 103 \\
8 & 93 & 92 & 92 & 92 & 91 & 89 \\
9 & 87 & 87 & 86 & 86 & 83 & 78 \\
10 & 83 & 83 & 82 & 81 & 77 & 69 \\
Total & 1000 & 1000 & 1000 & 1001 & 1000 & 1001 \\
\hline
\end{tabular}

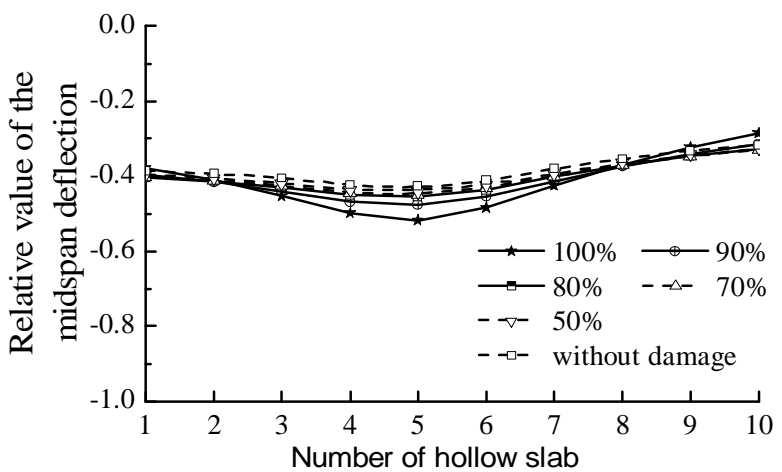

Figure 8 Extent of hinge joints damage-proportion of vertical deflection of void slabs 
It can be found from Figure 7 that deflection proportion does not change significantly when the extent of hinge joints damage increases from 0 to $80 \%$. When it reaches $80 \%$ to $100 \%$, the deflection proportion value of slab \#1 increases dramatically, showing the trend of single slab carrying the load.

It can be found from Table 4 that as the hinge joints damage increases, influence line values of slabs \#3 7 tend to increase. At the same time, the complete damage of hinge joints has large effect on the transverse distribution characteristics of bridges. Figure 8 shows the proportion of mid-span deflection of slabs when imposing unit load on the medium slabs.

\section{Result analysis}

Trend of effect of pavement layer thickness. Result analysis shows that when imposing load on side slab \#1, with the increase of the pavement layer thickness, influence line values and deflection of slabs \#1 4 show a decreasing trend. Those of slab \#1 reduce most obviously--the influence line value reduces by $2.5 \%$ and deflection $37.0 \%$ when the pavement layer thickness is $20 \mathrm{~cm}$ compared to $5 \mathrm{~cm}$, indicating that the increase of pavement layer thickness has limited effect on improving transverse load distribution of void slab bridge, but it can improve the overall stiffness, thus enhancing the bending resistance of the bridge; when imposing load on slab \#5, with the increase of the pavement layer thickness, influence line values and deflection of slabs \#4, \#5 and \#6 are reduced significantly. The influence line value of slab \#5 is reduced by about $0.8 \%$ and deflection $36.3 \%$; when imposing unit load on side slab and medium slab, in the influence pattern of pavement layer thickness on transverse distribution coefficient of void slab, load at side slab plays a major role. Increasing pavement layer thickness within a certain range can enhance the effect of transverse distribution of load and improve the overall stiffness of the bridge, thereby improving the mechanical properties of the simply supported void slab bridge.

Rules of hinge joints effect. Results show that when imposing load on side slab \#1, with the increase of hinge joints damage extent, influence line value and deflection of slabs \#1 4 tend to increase. Those of slab \#1 increase most obviously-influence line value increases by $19 \%$ and deflection increases by $24.9 \%$ when the hinge joints are completely destroyed compared to no damage, indicating that the extent of hinge joints damage has significant effect on transverse load distribution coefficient, because as the key parts of the void slab bridge, hinge joints bear complex stress, and transverse shear transfer between void slabs mainly rely on hinge joints, whose damage extent directly affects transverse load distribution; when imposing load on medium slab \#5, with the increase of hinge joints damage extent, influence line value and deflection of slabs \#3 7 tend to increase. The influence line value of slab \#5 increases by about $11.6 \%$ and deflection $17.6 \%$; when hinge joints is not seriously damaged, it has little effect on transverse load transfer, while when the extent of damage exceeds certain limit (as $80 \%$ herein), the effect increases rapidly. Therefore, maintaining the integrity of hinge joints is the most effective way to ensure multi-slabs loading of void slabs.

\section{Conclusions}

1) Increasing pavement layer thickness mainly improves bridge' $s$ bending strength, and enhances the effect of transverse load distribution to some extent. In exceptional circumstances, increasing pavement layer thickness can be used to improve transverse distribution characteristics of the bridge.

2) Extent of hinge joints damage directly affects the rule of transverse load distribution of the bridge, so to ensure the integrity of the hinge joints is the most effective way to improve bridge' s mechanical characteristics.

3) Hinge joints damage has a certain limit, and when the damage exceeds this limit, its adverse impact on bridge transverse load transfer increases sharply, so in actual engineering, to protect hinge joints from being completely destroyed is a key factor to ensure its normal function. 


\section{Acknowledgments}

This study is financially supported by the National Natural Science Foundation of China (Grant no.51468013 ), and the Science Research and Technology Development Project of Guangxi Zhuang Autonomous Region (Program, No.1355008-9).

\section{References}

[1] Q.W. Liu,C.M. Wang, J. Li:Comparison and analysis of calculation method of transverse load distribution for fabricated hollowed slab bridges. Journal of architecture and civil engineering,Vol.28(2011),p. 100-105

[2]G.H. Li,D. Shi: Calculation of transverse load distribution for highway bridges( china comunication press,Beijing 1987).

[3]Q. Wang, W.P. Hu:Method of design and calculation for slab bridge.highway, Vol.11(2009)p. 17-19.

[4]F. W. Xu: Study on transverse distribution of bridge vehicle interaction of simply supported plate girder bridge.Hangzhou: Zhejiang University,2006.

[5]G.L. Dai:The study of design and analysis on bridge structure and its application(china comunication press, . Beijing,2001).

[6]C.G. Liu :Study on design procedure of crosswise hinge joint for the assembly hollow slab girder bridge. Harbin:Harbin University of technology,2013. 